\title{
Characteristics of tuberculosis patients and the evaluation of compliance to the national TB management guidelines at clinics in a rural community from Mpumalanga province, South Africa
}

\author{
Jolly Musoke ${ }^{a *}$ and Anita L. Michel ${ }^{a}$ \\ ${ }^{a}$ Faculty of Veterinary Science, Department of Veterinary Tropical Diseases, Onderstepoort, South Africa \\ *Corresponding author, email:jolly.musoke@hotmail.com
}

This study serves as baseline investigation into tuberculosis (TB) patient population characteristics and the compliance of clinics in rural settings to the national TB guidelines in terms of diagnosing the disease. A total of 62 TB positive patients' files were reviewed. Patients were diagnosed using: smear microscopy (41.9\%); chest radiography (37.1\%); Xpert MTB/RIF (9.7\%); symptoms (3.2\%); abdomen sonar (1.6\%); and, no record (6.5\%). Lack of complete compliance was identified, including large dependencies on chest X-ray as the first line of diagnosis and inadequate diagnosis of extra-pulmonary TB. These findings could assist identifying health system gaps for provincial and national control programs.

Keywords: Tuberculosis, rural community, TB patient profiles, compliance to TB national guidelines

\section{Introduction}

Tuberculosis (TB) remains a global health crisis claiming 1.3 million lives and accounting for 8.6 million new cases reported in the year 2012. ${ }^{1}$ According to the World Health Organisation (WHO), South Africa is among the top three countries on the African continent bearing the highest TB burden, with a TB incidence of 1003 per 100000 population (2012). The South African Department of Health reported a lower TB incidence (all TB) of 687 per 100000 population (2012), and 62827 deaths (2011). Despite a discrepancy in the rates between the two reports, they both serve to highlight the alarming rates of TB in South Africa.

The South African national tuberculosis programs (e.g. Health System's Trust) and international health organisations (e.g. WHO) rely on obtaining critical data from TB registers compiled at provisional or national levels. Often these registers are incomplete and do not reflect the individual communities bearing the brunt of the disease. ${ }^{2}$ It is of importance to conduct community-level studies, profiling the population infected with TB and comparing with published reports. These studies are essential for targeted, appropriate and tailor-made control measures and interventions, particularly in resource-poor settings. Not all people who seek care at health facilities are correctly diagnosed and treated according to the national guidelines and recommendations. ${ }^{3}$

The aim of this study was to conduct a baseline study of TB patient population characteristics in a rural community setting. Investigations into whether the diagnostic techniques used in the rural community are in line with the current National Tuberculosis Control programme (NTP) with regards to diagnosing the disease were also conducted.

\section{Materials and methods \\ Study area and study population}

This study was conducted in a small rural community that forms part of the Mnisi tribal chieftainship, located in the north-eastern corner of Bushbuckridge municipal area, Mpumalanga province,
South Africa. The community was selected based on the geographical location, at the western border of the Greater Kruger National Park Complex (GKNPC) conservation area (Figure 1), at the wildlife/livestock/human/platform ('One health' research platform). The community is home to over 40000 , mainly Shangaan speaking people. The community has high levels of unemployment and a low family income is the main socio-economic indicator.

\section{Health services}

The study community's main health service providers are four government primary health care clinics and one referral hospital, Tintswalo. The referral hospital is a 423 bedded acute-care government hospital located $48 \mathrm{~km}$ from the study community. All sputum testing is performed by the National Health Laboratory Services in Bushbuckridge, while X-ray facilities are available at Tintswalo hospital.

The community is under the administration of Ehlanzeni district and, in 2010, the district had the highest number (12 459) of reported TB cases (all TB cases) in Mpumalanga province. ${ }^{4,5}$ The latest (2012) health survey reported a TB incidence of 630 per 100000 population in the district. ${ }^{5}$ The local TB control program is aligned with the World Health Organisation's Directly Observed Treatment Short-Course (DOTS) strategy.

\section{Data collection}

Between June 2013 and June 2014, a retrospective study was conducted at the four primary healthcare clinics, namely Hluvukani, Gotternburg, Welverdiend and Utah A (Figure 1). Patient demographic data and disease classification were extracted from the TB patient files enrolled at the four clinics during the study period (one year). Data from the patient files was directly entered into Microsoft Excel. The demographic data collected included: patient age; gender; and TB patient category; Disease classification included site of TB disease and diagnostic method. In this study, patient category and site of disease were defined as stated in the NTP. ${ }^{6}$ Briefly, new TB cases referred to patients who have never had anti-tuberculosis medication or to 


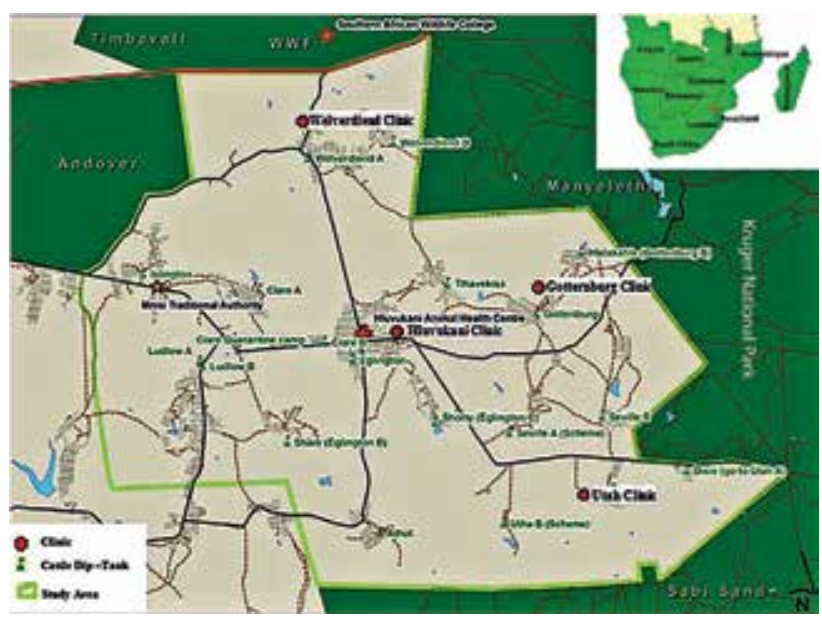

Figure 1: Geographical location of the health clinics in the Mnisi study site.

those who have taken TB treatment for less than four weeks. A defaulter TB case refers to a patient who has completed at least one month of TB treatment and returns after interrupting treatment for two or more months. Retreatment after failure defines patients with a positive smear or culture at the end of treatment. Multidrug resistant TB (MDR-TB) defines cases where tuberculosis isolates are resistant to at least isoniazid (INH) and rifampin (RIF). Extra-pulmonary TB is the spread/dissemination of TB in organs (e.g. pleura, lymph nodes or abdomen) other than the lungs.

\section{Data analysis}

Data in the Microsoft Excel spreadsheet was analysed using SAS software, version 9.2 of the SAS system for Windows.

\section{Ethics statement}

The study was carried out with ethical approval from the University of Pretoria, Faculty of Health Sciences Research Ethics Committee and from the Mpumalanga Provincial Government, Department of Health Provincial Research and Ethics Committee.

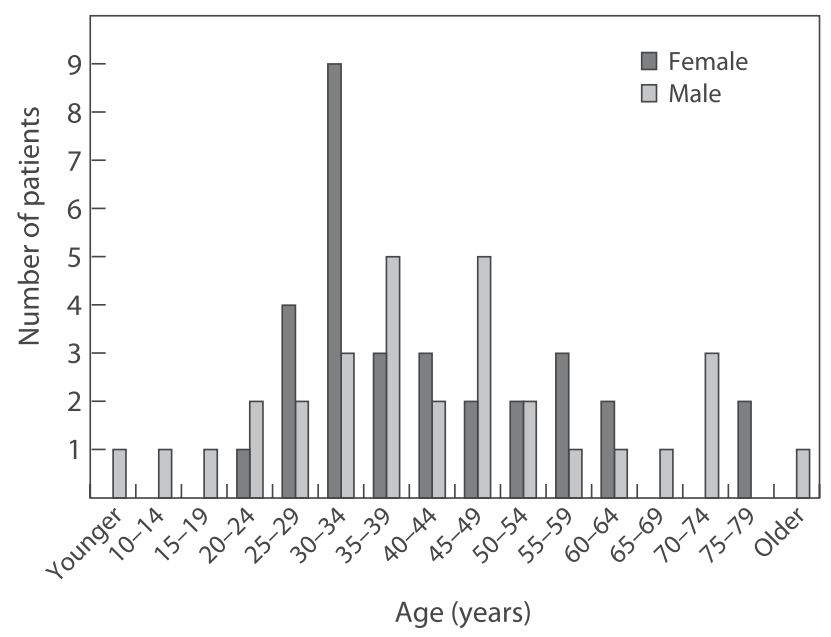

Figure 2: The age distribution of TB positive patients in the Mnisi community.

\section{Results}

\section{Health services}

A total of 62 TB patient files relating to TB positive patients were reviewed during the one-year duration of this study. Patients were diagnosed as TB-positive using the following techniques: smear microscopy, 41.9\% ( $n=26)$; chest radiography, $37.1 \%$ $(n=23)$; Xpert MTB/RIF assay, $9.7 \%(n=6)$; symptoms, $3.2 \%$ $(n=2)$; abdomen sonar, $1.6 \%(n=1)$; and, no record, $6.5 \%(n=4)$.

\section{Gender and age distribution}

The TB cases were equally distributed between males and females ( $n=31$ each).

Overall, the highest incidence of TB was observed in patients aged 30-34 years. In males, the age categories of 35-39 and 4549 years were mainly affected; whereas, in females, the highest impact of TB was observed in the age category 30-34 years (Figure 2).

A total of $87.1 \%(n=54)$ of the reviewed TB cases were classified as new cases; $6.5 \%(n=4)$ were classified as retreatment after default; $4.8 \%(n=3)$ were retreatment after failure cases. One case $(1.6 \%)$ was identified as a MDR-TB case.

Nine cases were classified as (14.5\%) extra-pulmonary TB; of these, two cases were adenitis, and two cases were TB of the pleura.

\section{Discussion}

From June 2013 to June 2014, a total of 62 TB patient files were retrieved from the four primary healthcare clinics. The majority of patients were diagnosed using smear microscopy (41.9\%), chest radiography (37.1\%), Xpert MTB/RIF (9.7\%) and no record (11.3\%). According to the NTP guidelines, diagnosis of TB depends, among other factors on the presentation of patients with TB symptoms, screening practices at the health facilities, and turnaround time for delivery on results. However, the tests used are to follow provided guidelines. Part of the South African TB control campaign was the introduction and rolling out of new diagnostic tools. One of the innovative techniques is Xpert MTB/ $\mathrm{RIF}$, a cartridge based automated diagnostic technique that detects DNA sequences specific for Mycobacterium tuberculosis complex and resistance to the antibiotic, rifampicin, using nucleic acid amplification. ${ }^{7}$ South Africa is the first high TB burden country to implement Xpert MTB/RIF in its government polices as the first diagnostic tool for all suspected TB cases. ${ }^{8}$ In the Mnisi community, it was observed that in more recent cases, the use of Xpert MTB/RIF had replaced smear microscopy as the first line of diagnosis. Therefore, in conclusion, the NTP components were in the process of being implemented, and the national roll-out implementation of the new diagnostic tool is beneficial to communities including those in rural areas.

However, it was observed that there was a great dependence on using chest X-rays (37.1\%) as the first line of diagnosis, especially in patients transferred from the referral Tintswalo hospital to the clinics. This is contradictory to the NTP policies as the report states that chest $X$-rays should not be used as the only diagnostic test. Chest X-rays are to be used in patients who cannot produce sputum, patients with negative Xpert MTB/RIF results and who are HIV positive, or in patients with extra-pulmonary TB. The $\mathrm{X}$-ray findings are to be interpreted with the patient's history and clinical findings. ${ }^{6}$ Similar findings on the over-reliance of chest $X$-rays for diagnosis of TB has been reported in a case study in Durban, were $45 \%$ of TB cases were diagnosed on the basis of a chest $\mathrm{X}$-ray. ${ }^{3}$ This finding highlights the urgent need for the 
complete implementation of all components of the NTP within the study community and supporting health systems.

In Mpumalanga province in 2010, 10\% (2 331) of reported TB cases were extra-pulmonary. A slightly higher percentage of extra-pulmonary TB cases were observed in the Mnisi community, at $14.5 \%(n=9)$. Complete details of these cases and site of infection were not available from the TB patient files and TB register. However, the few files which were classified as extrapulmonary TB were adenitis $(n=2)$, and TB of the pleura $(n=2)$. Extra-pulmonary TB is highly elusive in detection and requires clear and complete communication in the health system in order to administer timely and appropriate chemotherapy. In addition, the NTP guidelines (2014) state that patients suspected of extrapulmonary $\mathrm{TB}$ are to have specimens taken from the site and Xpert MTB/RIF, culture and drug susceptibility testing performed. However, in the cases of the Mnisi patient files this protocol had not been followed. The lack of a complete set of information flags the severe constraint in diagnosing extra-pulmonary TB patients, and the urgency to raise awareness among health providers on the importance of complete investigation and reporting according to the NTP guidelines.

The age category of 30-34 years experienced the greatest impact from TB. These findings follow national trends reported in recent years in which TB has been more common in younger generations, particularly males in their 30's and women in their $20^{\prime} s .{ }^{9}$ The age trend is largely attributed to the high rise of human immunodeficiency virus (HIV). Lawn and co-workers (2006) reported a 2.5-fold increase of TB notification rates as the rise of HIV prevalence increased (1996-2004), particularly observed in the age category of $20-39$ years. $^{2}$

In the Mnisi community, $87.1 \%(n=54)$ TB cases were classified as new cases; $6.5 \%(n=4)$ were retreatment after default; $4.8 \%$ $(n=3)$ were retreatment after failure. One case $(1.6 \%)$ was identified as a MDR-TB case. These results are in accordance with countrywide findings as similar trends had been reported nationally, classifying the majority (85.0\%) of TB cases as new; 7.6\% relapse; $0.9 \%$ treatment failure; $2.2 \%$ treatment after default; and, $4.3 \%$ other. ${ }^{1}$ However, the low detection of MDR in the study community could possibly be underestimated as the improved Xpert MTB/RIF assay was only introduced in the clinics at a later stage during the study. The high number of new TB cases reflects high TB transmission rates. ${ }^{10}$ High contact rates, low case finding, delays in diagnosis, resource-limited health systems, and delays in chemotherapy are among the factors contributing to the high TB transmission rates. To achieve long term control of TB, reduction of the transmission rates should be one of the main priorities.

\section{Limitations}

The main limitation of this study was the availability of complete population data for the Mnisi community, including patients referred to Tintswalo hospital. Tintswalo hospital was not included in this study as it did not form part of the study area and associated research approval, hence no access to hospital data was possible. The limited data prohibited analysis of TB prevalence within the Mnisi community. The TB register file and patient files were partially incomplete and paper-based, which compromised tracing of patients. The HIV status of the study population could not be accessed and analysed due to ethical considerations, thus limiting access to patient information within the scope of this study (i.e. TB).

\section{Conclusion}

From this study in a rural setting, it became evident that the basic components of the NTP guidelines were implemented at rural clinic level. However, there is a need for implementation and awareness of all the components. This includes less dependence on chest $\mathrm{X}$-rays for diagnosis and improved diagnosis of extrapulmonary TB.

This study highlights the importance of community-based studies in providing baseline data which can help to identify health system gaps for provincial and national TB control programs.

Conflict of interest - The authors declare that no conflict of interest exists.

Funding - This study was funded by the Institute of Tropical Medicine in Antwerp, Belgium (ITM) through a framework agreement with the Belgian Development Cooperation (DGD) and South African National Parks.

Acknowledgements - We would like to thank the Mpumalanga Health Services, as well as the Mnisi Health Clinics nurses and all staff for their collaboration, assistance and communication with patients for the purpose of this study. We also thank Jaqui Somerville and Rene Ehlers for their assistance with the statistical analysis; and, Thuli Anastasia Maabane for her assistance in the field. We are thankful to Ongole Joven Jebio and Ravi Raveendranathan for assistance in editing the manuscript.

\section{References}

1. World Health Organization. Global tuberculosis report; 2013 [cited 2015 Jan 23]. Available from http://apps.who.int/iris/ bitstream/10665/91355/1/9789241564656_eng.pdf

2. Lawn SD, Bekker LG, Middelkoop K, et al. Impact of HIV infection on the epidemiology of tuberculosis in a peri-urban community in South Africa: The need for age-specific interventions. Clin Infect Dis. 2006;42:1040-47. doi:10.1086/501018.

3. Loveday $M$, Thomson $L$, Ndlela $Z$, et al. The implementation of the National Tuberculosis Control Programme (NTCP) at a regional/ district hospital and three of its feeder clinics: A Case Study. In: Systems Health, editor. National Department of Health. Trust: Pretoria; 2007;24-32.

4. Ehlanzeni District. Ehlanzeni District Municipality final report IDP 2012/13 -2015/16. 1-374 (2013)

5. Loveday M. Tuberculosis Indicator Comparisons by District. In: Massyn N, et al., editors. District Health Barometer 2012/13. Health Systems Trust; 2013. p. 179-201.

6. TB DOTS Strategy Coordination, National Department of Health Fishwicks Pretoria, 2014;p. 173.

7. Boehme CC, Nabeta P, Hillemann D, et al. Rapid Molecular Detection of Tuberculosis and Rifampin Resistance. N Engl J Med. 2010;363:100515. doi:10.1056/NEJMoa0907847

8. World Health Organization. Countdown to 2015 Global Tuberculosis Report. 2013 [cited 2013 Nov 20]. Supplement. Available from http://apps.who.int/iris/bitstream/10665/91542/1/WHO_HTM_ TB_2013.13_eng.pdf

9. Karim SSA, Churchyard GJ, Karim QA, et al. HIV infection and tuberculosis in South Africa: an urgent need to escalate the public health response. Lancet. 2009;374:921-33. doi:10.1016/S01406736(09)60916-8.

10. Wood R, Lawn SD, Johnstone-Robertson S, Bekker LG. Tuberculosis control has failed in South Africa - time to reappraise strategy. S Afr Med J. 2011;101:111-4. 